\title{
An Alternative Proof for the Capacity Region of the Degraded Gaussian MIMO Broadcast Channel
}

\author{
Ersen Ekrem, Student Member, IEEE, and Sennur Ulukus, Member, IEEE
}

\begin{abstract}
We provide an alternative proof for the capacity region of the degraded Gaussian multiple-input multiple-output (MIMO) broadcast channel. Our proof does not use the channel enhancement technique as opposed to the original proof of Weingertan et al. and the alternative proof of Liu et al. Our proof starts with the single-letter description of the capacity region of the degraded broadcast channel, and by using it, obtains a tight (i.e., achievable) outer bound for the capacity region of the degraded Gaussian MIMO broadcast channel, by using two main technical tools. The first one is the generalized de Bruijn identity due to Palomar et al. which provides a connection between the differential entropy and the Fisher information matrix. The second tool we use is an inequality due to Dembo which lower bounds the differential entropy in terms of the Fisher information matrix.
\end{abstract}

Index Terms - Capacity region, de Bruijn identity, Fisher information matrix, Gaussian MIMO broadcast channel.

\section{INTRODUCTION}

$\mathbf{T}$ HE GAUSSIAN multiple-input multiple-output (MIMO) broadcast channel consists of one transmitter and an arbitrary number of receivers, where the transmitter and receivers are equipped with multiple antennas. In this channel, each link between the transmitter and each receiver is modeled by a linear additive Gaussian channel. In general, the Gaussian MIMO broadcast channel is nondegraded, thus, we do not have a single-letter description of the capacity region. Despite this lack of a single-letter description, the capacity region of the Gaussian MIMO broadcast channel is successfully obtained in [1]. Subsequently, an alternative proof is given in [2]. In both proofs, the channel enhancement technique [1] is the main tool.

Reference [1] obtains the capacity region of the Gaussian MIMO broadcast channel in three main steps. As the first step, [1] finds the capacity region of the degraded Gaussian MIMO broadcast channel. To this end, [1] first shows that as opposed to the scalar Gaussian broadcast channel [3], the entropy-power inequality falls short of providing a converse proof for the degraded Gaussian vector, i.e., MIMO, broadcast channel. This insufficiency of the entropy-power inequality is alleviated by the invention of the channel enhancement technique [1]. Using this technique, [1] constructs a new degraded Gaussian

Manuscript received February 19, 2010; revised July 27, 2011; accepted October 30, 2011. Date of current version March 13, 2012. This work was supported by the National Science Foundation under Grants CCF 04-47613, CCF 05-14846, CNS 07-16311, and CCF 07-29127. The material in this paper was presented in part at the 2010 IEEE Information Theory Workshop.

The authors are with the Department of Electrical and Computer Engineering, University of Maryland, College Park, MD 20742 USA (e-mail: ersen@umd. edu; ulukus@umd.edu).

Communicated by S. N. Diggavi, Associate Editor for Shannon Theory.

Digital Object Identifier 10.1109/TIT.2011.2177557
MIMO broadcast channel for each point on the boundary of the Gaussian rate region ${ }^{1}$ of the original degraded channel, where the boundaries of the Gaussian rate regions of both channels intersect at that point, and the capacity region of the constructed degraded channel includes the capacity region of the original one. Then, [1] completes the first step of the proof by showing that the Gaussian rate region is the capacity region of the constructed degraded channel, for which Bergmans' converse [3] can be adapted as opposed to the original channel.

Secondly, [1] considers the aligned Gaussian MIMO broadcast channel, where the transmitter and all receivers have the same number of antennas. This channel is not degraded, thus, there is no single-letter expression for its capacity region. Reference [1] shows that the achievable rate region obtained by using dirty paper coding (DPC), i.e., the DPC region, is the capacity region of the aligned channel. For this purpose, [1] uses the channel enhancement technique one more time along with the capacity result obtained for the degraded Gaussian MIMO broadcast channel in the first step.

Finally, [1] considers the general, not necessarily degraded or aligned, Gaussian MIMO broadcast channel and shows that the DPC region again amounts to the capacity region by using some limiting arguments in conjunction with the capacity result obtained for the aligned channel.

Similar to the proof of [1], the alternative proof in [2] uses the channel enhancement technique as well. The alternative proof in [2] can be divided into two parts. In the first part, [2] considers an optimization problem which is the maximization of the difference of two differential entropy terms (see [2, Theorem 1]) which cannot be solved by a stand-alone use of the entropy-power inequality. Next, [2] provides two proofs for the fact that the Gaussian distribution is the maximizer of this optimization problem. In both proofs provided in [2], the channel enhancement technique is used. In the second part, [2] considers Marton's outer bound [4] for the general broadcast channel, and evaluates it for the aligned Gaussian MIMO broadcast channel by using the optimization problem solved in the first part. This evaluation yields the capacity region of the two-user aligned Gaussian MIMO broadcast channel.

We note that though the proof in [2] is for the aligned, not necessarily degraded, Gaussian MIMO broadcast channel, if this proof is adapted to find the capacity region of the degraded Gaussian MIMO broadcast channel, again the channel enhancement technique will be needed. In particular, for the degraded case, the optimization problem solved in the first part of the proof in [2] will change slightly, however the need for channel

\footnotetext{
${ }^{1}$ The Gaussian rate region refers to the achievable rate region obtained by superposition coding and successive decoding with Gaussian codebooks.
} 
enhancement will remain. In fact, the optimization problem needed for the degraded case is a special case of the original optimization problem solved in the first part of the proof in [2], which is given in [2, Corollary 4].

Here, we revisit the degraded Gaussian MIMO broadcast channel and provide an alternative proof for the capacity region of this degraded channel, without using the channel enhancement technique. Though channel enhancement is an elegant technique that finds itself diverse applications, we believe that our proof is more direct. On the other hand, our proof is limited to the degraded case and does not seem to be extendable for the general case. In other words, to obtain the capacity region for the general case after finding the capacity region for the degraded case through our proof, one needs to use the channel enhancement technique [1].

Our proof starts with the single-letter description of the capacity region of the degraded broadcast channel, and by using it, obtains a tight (i.e., achievable) outer bound for the capacity region of the degraded Gaussian MIMO broadcast channel, by using two main technical tools. The first one is the generalized de Bruijn identity due to [5] that states a connection between the differential entropy and the Fisher information matrix. The second tool we use is an inequality due to [6], [7] that gives a lower bound for the differential entropy in terms of the Fisher information matrix.

Finally, our technique used in this alternative proof can be useful in other vector Gaussian multiterminal information theory problems when proving the optimality of Gaussian random vectors. In fact, we have used a variant of this technique in proving the secrecy capacity region of the Gaussian MIMO multireceiver wiretap channel in [8], and the secrecy capacity region of the Gaussian MIMO degraded compound multireceiver wiretap channel in [9].

\section{Channel Model and Main Result}

The (aligned) degraded $K$-user Gaussian MIMO broadcast channel is defined by

$$
\mathbf{Y}_{k}=\mathbf{X}+\mathbf{N}_{k}, \quad k=1, \ldots, K
$$

where $\mathbf{N}_{k}$ is Gaussian with covariance matrix $\boldsymbol{\Sigma}_{k}, k=$ $1, \ldots, K$ and the channel input $\mathbf{X}$ and outputs $\left\{\mathbf{Y}_{k}\right\}_{k=1}^{K}$ satisfy the Markov chain

$$
\mathbf{X} \rightarrow \mathbf{Y}_{1} \rightarrow \ldots \rightarrow \mathbf{Y}_{K}
$$

which is equivalent to the covariance matrices $\left\{\boldsymbol{\Sigma}_{k}\right\}_{k=1}^{K}$ satisfying the following order:

$$
\mathbf{0} \prec \Sigma_{1} \preceq \ldots \preceq \Sigma_{K} .
$$

The channel input is subject to a covariance constraint

$$
E\left[\mathbf{X X}^{\top}\right] \preceq \mathbf{S}
$$

where we assume $\mathbf{S} \succ \mathbf{0}$. The covariance constraint in (4) is more general than many other constraints including the trace constraint, in the sense that, once the capacity region is found for the constraint in (4), capacity regions arising from the use of other constraints subsumed by (4) can be obtained by using this capacity region [1].

We next note that the definition of degradedness can be generalized to the case where receivers get arbitrary linear combinations of the channel inputs, i.e.,

$$
\mathbf{Y}_{k}=\mathbf{H}_{k} \mathbf{X}+\mathbf{N}_{k}, \quad k=1, \ldots, K .
$$

The broadcast channel defined in (5) is said to be degraded, i.e., satisfies the Markov chain in (2), if there exist matrices $\left\{\mathbf{D}_{k}\right\}_{k=1}^{K-1}$ such that $\mathbf{D}_{k} \mathbf{H}_{k}=\mathbf{H}_{k+1}$ and $\mathbf{D}_{k} \mathbf{D}_{k}^{\top} \preceq \mathbf{I}[10]$. However, once the capacity region of the aligned degraded Gaussian MIMO broadcast channel defined by (1) is obtained, the capacity region of the general degraded Gaussian MIMO broadcast channel defined by (5) can be obtained by following the analysis given in Section V of [10], which essentially relies on some limiting arguments. Since the key step to obtain the capacity region of the general degraded Gaussian MIMO broadcast channel defined by (5) is to establish the capacity region of the aligned degraded Gaussian MIMO broadcast channel defined by (1), here we consider only the latter channel model.

The capacity region of the Gaussian MIMO broadcast channel is established in [1] for the most general case. For the degraded case, it is given as follows.

Theorem 1 ([1, Theorem 2]): The capacity region of the $K$-user degraded Gaussian MIMO broadcast channel is given by the union of rate tuples $\left(R_{1}, \ldots, R_{K}\right)$ satisfying

$$
R_{k} \leq \frac{1}{2} \log \frac{\left|\sum_{i=1}^{k} \mathbf{K}_{i}+\boldsymbol{\Sigma}_{k}\right|}{\left|\sum_{i=1}^{k-1} \mathbf{K}_{i}+\mathbf{\Sigma}_{k}\right|}
$$

where the union is over all positive semi-definite matrices $\left\{\mathbf{K}_{i}\right\}_{i=1}^{K}$ such that $\sum_{i=1}^{K} \mathbf{K}_{i}=\mathbf{S}$.

In the next section, we provide an alternative proof for this theorem for $K=2$, and in Section IV we extend this proof to the case $K>2$. In both cases, we use the capacity region of the degraded broadcast channel which is stated in the following theorem, for the Gaussian MIMO channel at hand.

Theorem 2 ([11, Theorem 15.6.2]): The capacity region of the degraded broadcast channel is given by the union of rate tuples $\left(R_{1}, \ldots, R_{K}\right)$ satisfying

$$
R_{k} \leq I\left(U_{k} ; Y_{k} \mid U_{k+1}\right), \quad k=1, \ldots, K
$$

where $U_{K+1}=\phi, U_{1}=X$, and the union is over all $\left\{U_{k}\right\}_{k=2}^{K}, X$ such that

$$
U_{K} \rightarrow \ldots \rightarrow U_{2} \rightarrow X \rightarrow Y_{1} \rightarrow \ldots \rightarrow Y_{K}
$$

\section{Proof OF THEOREM 1 FOR $K=2$}

\section{A. Background}

We need some properties of the Fisher information and the differential entropy, which are provided next. 
Definition 1([8, Definition 3]): Let $(\mathbf{U}, \mathbf{X})$ be an arbitrarily correlated length- $n$ random vector pair with well-defined densities. The conditional Fisher information matrix of $\mathbf{X}$ given $\mathbf{U}$ is defined as

$$
\mathbf{J}(\mathbf{X} \mid \mathbf{U})=E\left[\boldsymbol{\rho}(\mathbf{X} \mid \mathbf{U}) \boldsymbol{\rho}(\mathbf{X} \mid \mathbf{U})^{\top}\right]
$$

where the expectation is over the joint density $f(\mathbf{u}, \mathbf{x})$, and the conditional score function $\boldsymbol{\rho}(\mathbf{x} \mid \mathbf{u})$ is

$$
\begin{aligned}
\boldsymbol{\rho}(\mathbf{x} \mid \mathbf{u}) & =\nabla \log f(\mathbf{x} \mid \mathbf{u}) \\
& =\left[\frac{\partial \log f(\mathbf{x} \mid \mathbf{u})}{\partial x_{1}} \ldots \frac{\partial \log f(\mathbf{x} \mid \mathbf{u})}{\partial x_{n}}\right]^{\top} .
\end{aligned}
$$

We first present the conditional form of the Cramer-Rao inequality, which is proved in [8].

Lemma 1 ([8, Lemma 13]): Let $\mathbf{U}, \mathbf{X}$ be arbitrarily correlated random vectors with well-defined densities. Let the conditional covariance matrix of $\mathbf{X}$ be $\operatorname{Cov}(\mathbf{X} \mid \mathbf{U}) \succ \mathbf{0}$, then we have

$$
\mathbf{J}(\mathbf{X} \mid \mathbf{U}) \succeq \operatorname{Cov}(\mathbf{X} \mid \mathbf{U})^{-1}
$$

which is satisfied with equality if $(\mathbf{U}, \mathbf{X})$ is jointly Gaussian with conditional covariance matrix $\operatorname{Cov}(\mathbf{X} \mid \mathbf{U})$.

The following lemma will be used in the upcoming proof. The unconditional version of this lemma, i.e., the case $\mathbf{T}=\phi$, is proved in [8, Lemma 6].

Lemma 2 ([8, Lemma 6]): Let $\mathbf{T}, \mathbf{U}, \mathbf{V}_{1}, \mathbf{V}_{2}$ be random vectors such that $(\mathbf{T}, \mathbf{U})$ and $\left(\mathbf{V}_{1}, \mathbf{V}_{2}\right)$ are independent. Moreover, let $\mathbf{V}_{1}, \mathbf{V}_{2}$ be Gaussian random vectors with covariance matrices $\boldsymbol{\Sigma}_{1}, \boldsymbol{\Sigma}_{2}$ such that $\mathbf{0} \prec \boldsymbol{\Sigma}_{1} \preceq \boldsymbol{\Sigma}_{2}$. Then, we have

$$
\mathbf{J}^{-1}\left(\mathbf{U}+\mathbf{V}_{2} \mid \mathbf{T}\right)-\boldsymbol{\Sigma}_{2} \succeq \mathbf{J}^{-1}\left(\mathbf{U}+\mathbf{V}_{1} \mid \mathbf{T}\right)-\boldsymbol{\Sigma}_{1}
$$

The following lemma will also be used in the upcoming proof.

Lemma 3 ([8, Lemma 8]): Let $\mathbf{K}_{1}, \mathbf{K}_{2}$ be positive semi-definite matrices satisfying $\mathbf{0} \preceq \mathbf{K}_{1} \preceq \mathbf{K}_{2}$, and $\mathbf{f}(\mathbf{K})$ be a matrix-valued function such that $\mathbf{f}(\mathbf{K}) \succeq \mathbf{0}$ for $\mathbf{K}_{1} \preceq \mathbf{K} \preceq \mathbf{K}_{2}$. Moreover, $\mathbf{f}(\mathbf{K})$ is assumed to be gradient of some scalar field. Then, we have

$$
\int_{\mathbf{K}_{1}}^{\mathbf{K}_{2}} \mathbf{f}(\mathbf{K}) d \mathbf{K} \geq 0
$$

The following generalization of the de Bruijn identity [12], [13] is due to [5], where the unconditional form of this identity, i.e., $\mathbf{U}=\phi$, is proved. Its generalization to this conditional form for an arbitrary $\mathbf{U}$ is rather straightforward, and is given in $[8$, Lemma 16].

Lemma 4 ([8, Lemma 16]): Let $(\mathbf{U}, \mathbf{X})$ be an arbitrarily correlated random vector pair with finite second order moments, and also be independent of the random vector $\mathbf{N}$ which is zeromean Gaussian with covariance matrix $\boldsymbol{\Sigma}_{N} \succ \mathbf{0}$. Then, we have

$$
\nabla_{\boldsymbol{\Sigma}_{N}} h(\mathbf{X}+\mathbf{N} \mid \mathbf{U})=\frac{1}{2} \mathbf{J}(\mathbf{X}+\mathbf{N} \mid \mathbf{U}) .
$$

The following lemma is due to [6], [7] which lower bounds the differential entropy in terms of the Fisher information matrix.

Lemma 5 ([6], [7]): Let $(U, \mathbf{X})$ be an $(n+1)$-dimensional random vector, where the conditional Fisher information matrix of $\mathbf{X}$, conditioned on $U$, exists. Then, we have

$$
h(\mathbf{X} \mid U) \geq \frac{1}{2} \log (2 \pi e)^{n}\left|\mathbf{J}^{-1}(\mathbf{X} \mid U)\right| .
$$

In [6] and [7], the unconditional version of this lemma, i.e., $U=\phi$, is provided. A proof for its generalization to this conditional form is given in Appendix I.

\section{B. Prooffor $K=2$}

We first rewrite the capacity region of the degraded broadcast channel given in Theorem 2 for two users as a union of rate pairs $\left(R_{1}, R_{2}\right)$ satisfying

$$
\begin{aligned}
& R_{1} \leq I\left(X ; Y_{1} \mid U\right) \\
& R_{2} \leq I\left(U ; Y_{2}\right)
\end{aligned}
$$

where we dropped the subscript of the auxiliary random variable $U_{2}$ and denoted it simply as $U$. The involved random variables satisfy the Markov chain $U \rightarrow X \rightarrow Y_{1} \rightarrow Y_{2}$. To obtain the capacity region of the degraded Gaussian MIMO broadcast channel, we need to evaluate this region. In particular, we will show that the optimal random vector $(U, \mathbf{X})$ that exhausts this region is Gaussian, and the corresponding capacity region is given by the union of rate pairs $\left(R_{1}, R_{2}\right)$ satisfying

$$
\begin{aligned}
& R_{1} \leq \frac{1}{2} \log \frac{\left|\mathbf{K}+\boldsymbol{\Sigma}_{1}\right|}{\left|\boldsymbol{\Sigma}_{1}\right|} \\
& R_{2} \leq \frac{1}{2} \log \frac{\left|\mathbf{S}+\boldsymbol{\Sigma}_{2}\right|}{\left|\mathbf{K}+\boldsymbol{\Sigma}_{2}\right|}
\end{aligned}
$$

where the union is over all $\mathbf{K}$ such that $\mathbf{0} \preceq \mathbf{K} \preceq \mathbf{S}$. We note that the region described by (19)-(20) comes from Theorem 1 by dropping the subscript of $\mathbf{K}_{1}$ and denoting it simply as $\mathbf{K}$.

We begin with the bound on $R_{2}$. Starting from (18), we get

$$
\begin{aligned}
R_{2} & \leq I\left(U ; \mathbf{Y}_{2}\right) \\
& =h\left(\mathbf{Y}_{2}\right)-h\left(\mathbf{Y}_{2} \mid U\right) \\
& \leq \frac{1}{2} \log (2 \pi e)^{n}\left|\mathbf{S}+\boldsymbol{\Sigma}_{2}\right|-h\left(\mathbf{Y}_{2} \mid U\right)
\end{aligned}
$$

where the inequality in (23) comes from the maximum entropy theorem [11]. We now bound $h\left(\mathbf{Y}_{2} \mid U\right)$ in (23). We first get an upper bound as

$$
h\left(\mathbf{Y}_{2} \mid U\right) \leq h\left(\mathbf{Y}_{2}\right) \leq \frac{1}{2} \log (2 \pi e)^{n}\left|\mathbf{S}+\boldsymbol{\Sigma}_{2}\right|
$$

where the first inequality comes from the fact that conditioning cannot increase entropy, and the second inequality is due to the maximum entropy theorem [11]. Furthermore, using Lemma 5, we can get the following lower bound for $h\left(\mathbf{Y}_{2} \mid U\right)$

$$
h\left(\mathbf{Y}_{2} \mid U\right) \geq \frac{1}{2} \log (2 \pi e)^{n}\left|\mathbf{J}^{-1}\left(\mathbf{X}+\mathbf{N}_{2} \mid U\right)\right| .
$$


We next define the following function:

$$
r(t)=\frac{1}{2} \log (2 \pi e)^{n}\left|\mathbf{A}(t)+\boldsymbol{\Sigma}_{2}\right|, \quad 0 \leq t \leq 1
$$

where $\mathbf{A}(t)$ is given as

$$
\mathbf{A}(t)=(1-t)\left[\mathbf{J}^{-1}\left(\mathbf{X}+\mathbf{N}_{2} \mid U\right)-\mathbf{\Sigma}_{2}\right]+t \mathbf{S} .
$$

We first note that

$$
\begin{aligned}
\mathbf{J}^{-1}\left(\mathbf{X}+\mathbf{N}_{2} \mid U\right)-\boldsymbol{\Sigma}_{2} & \preceq \operatorname{Cov}\left(\mathbf{X}+\mathbf{N}_{2} \mid U\right)-\mathbf{\Sigma}_{2} \\
& =\operatorname{Cov}(\mathbf{X} \mid U) \\
& \preceq \operatorname{Cov}(\mathbf{X}) \\
& \preceq \mathbf{S}
\end{aligned}
$$

where (28) is a consequence of Lemma 1, and (30) comes from the fact that the conditional covariance matrix is smaller than the unconditional one in the positive semi-definite ordering sense. This implies that for any $0 \leq t \leq 1, \mathbf{A}(t)$ satisfies

$$
\mathbf{J}^{-1}\left(\mathbf{X}+\mathbf{N}_{2} \mid U\right)-\mathbf{\Sigma}_{2} \preceq \mathbf{A}(t) \preceq \mathbf{S} .
$$

Using $r(t)$, bounds in (24) and (25) can be rewritten as

$$
r(0) \leq h\left(\mathbf{Y}_{2} \mid U\right) \leq r(1) .
$$

Since $r(t)$ is continuous in $t$ [8], due to the intermediate value theorem, there exists a $t^{*}$ such that

$$
r\left(t^{*}\right)=h\left(\mathbf{Y}_{2} \mid U\right)=\frac{1}{2} \log (2 \pi e)^{n}\left|\mathbf{A}\left(t^{*}\right)+\boldsymbol{\Sigma}_{2}\right|
$$

where $\mathbf{A}\left(t^{*}\right)$ satisfies (32). Plugging (34) into (23) yields

$$
R_{2} \leq \frac{1}{2} \log \frac{\left|\mathbf{S}+\boldsymbol{\Sigma}_{2}\right|}{\left|\mathbf{A}\left(t^{*}\right)+\Sigma_{2}\right|}
$$

which is the desired bound on $R_{2}$ given in (20).

We now obtain the desired bound on $R_{1}$. To this end, using (32) and Lemma 2, we get

$$
\begin{aligned}
\mathbf{A}\left(t^{*}\right) & \succeq \mathbf{J}^{-1}\left(\mathbf{X}+\mathbf{N}_{2} \mid U\right)-\boldsymbol{\Sigma}_{2} \\
& \succeq \mathbf{J}^{-1}(\mathbf{X}+\mathbf{N} \mid U)-\mathbf{\Sigma}_{N}
\end{aligned}
$$

for any Gaussian random vector $\mathbf{N}$ with covariance matrix $\boldsymbol{\Sigma}_{N}$ where $\Sigma_{N} \preceq \Sigma_{2}$. The order in (37) is equivalent to

$$
\left(\mathbf{A}\left(t^{*}\right)+\boldsymbol{\Sigma}_{N}\right)^{-1} \preceq \mathbf{J}(\mathbf{X}+\mathbf{N} \mid U) .
$$

Next, we consider the bound on $R_{1}$ given by (17). To this end, we first find an upper bound for the differential entropy term $h\left(\mathbf{Y}_{1} \mid U\right)$ which will be subsequently used to obtain the desired bound on $R_{1}$.

$$
\begin{aligned}
& h\left(\mathbf{Y}_{1} \mid U\right)=h\left(\mathbf{Y}_{1} \mid U\right)-h\left(\mathbf{Y}_{2} \mid U\right)+h\left(\mathbf{Y}_{2} \mid U\right) \\
&= h\left(\mathbf{Y}_{1} \mid U\right)-h\left(\mathbf{Y}_{2} \mid U\right)+\frac{1}{2} \log (2 \pi e)^{n}\left|\mathbf{A}\left(t^{*}\right)+\boldsymbol{\Sigma}_{2}\right| \\
&=-\frac{1}{2} \int_{\boldsymbol{\Sigma}_{1}}^{\boldsymbol{\Sigma}_{2}} \mathbf{J}(\mathbf{X}+\mathbf{N} \mid U) d \boldsymbol{\Sigma}_{N}+\frac{1}{2} \log (2 \pi e)^{n}\left|\mathbf{A}\left(t^{*}\right)+\boldsymbol{\Sigma}_{2}\right| \\
& \leq-\frac{1}{2} \int_{\boldsymbol{\Sigma}_{1}}^{\boldsymbol{\Sigma}_{2}}\left(\mathbf{A}\left(t^{*}\right)+\boldsymbol{\Sigma}_{N}\right)^{-1} d \boldsymbol{\Sigma}_{N} \\
&+\frac{1}{2} \log (2 \pi e)^{n}\left|\mathbf{A}\left(t^{*}\right)+\boldsymbol{\Sigma}_{2}\right|
\end{aligned}
$$

$$
\begin{aligned}
& =\frac{1}{2} \log \frac{\left|\mathbf{A}\left(t^{*}\right)+\boldsymbol{\Sigma}_{1}\right|}{\left|\mathbf{A}\left(t^{*}\right)+\boldsymbol{\Sigma}_{2}\right|}+\frac{1}{2} \log (2 \pi e)^{n}\left|\mathbf{A}\left(t^{*}\right)+\boldsymbol{\Sigma}_{2}\right| \\
& =\frac{1}{2} \log (2 \pi e)^{n}\left|\mathbf{A}\left(t^{*}\right)+\boldsymbol{\Sigma}_{1}\right|
\end{aligned}
$$

where (40) is due to (34), (41) is obtained by using Lemma 4, and (42) is due to (38) and Lemma 3. Using (44) in (17), we get

$$
\begin{aligned}
R_{1} & \leq I\left(\mathbf{X} ; \mathbf{Y}_{1} \mid U\right) \\
& =h\left(\mathbf{Y}_{1} \mid U\right)-\frac{1}{2} \log (2 \pi e)^{n}\left|\mathbf{\Sigma}_{1}\right| \\
& \leq \frac{1}{2} \log \frac{\left|\mathbf{A}\left(t^{*}\right)+\boldsymbol{\Sigma}_{1}\right|}{\left|\boldsymbol{\Sigma}_{1}\right|}
\end{aligned}
$$

which is the desired bound on $R_{1}$ given in (19); completing the proof.

\section{Extension to the $K$-User CASE}

We now extend our alternative proof presented in the previous section to the case $K>2$. For that purpose, we need the following lemma due to [8] in addition to the tools introduced in Section III-A.

Lemma 6 ([8, Lemma 17]): Let $(\mathbf{V}, \mathbf{U}, \mathbf{X})$ be length- $n$ random vectors with well-defined densities. Moreover, assume that the partial derivatives of $f(\mathbf{u} \mid \mathbf{x}, \mathbf{v})$ with respect to $x_{i}, i=1, \ldots, n$ exist and satisfy

$$
\max _{1 \leq i \leq n}\left|\frac{\partial f(\mathbf{u} \mid \mathbf{v}, \mathbf{x})}{\partial x_{i}}\right| \leq g(\mathbf{u})
$$

for some integrable function $g(\mathbf{u})$. Then, if $(\mathbf{V}, \mathbf{U}, \mathbf{X})$ satisfy the Markov chain $\mathbf{V} \rightarrow \mathbf{U} \rightarrow \mathbf{X}$, we have

$$
\mathbf{J}(\mathbf{X} \mid \mathbf{U}) \succeq \mathbf{J}(\mathbf{X} \mid \mathbf{V}) .
$$

First, following the proof in Section III-B, we can show the existence of a covariance matrix $\mathbf{A}_{K}$ such that

$$
\begin{gathered}
\mathbf{J}^{-1}\left(\mathbf{X}+\mathbf{N}_{K} \mid U_{K}\right)-\boldsymbol{\Sigma}_{K} \preceq \mathbf{A}_{K} \preceq \mathbf{S} \\
h\left(\mathbf{Y}_{K} \mid U_{K}\right)=\frac{1}{2} \log (2 \pi e)^{n}\left|\mathbf{A}_{K}+\boldsymbol{\Sigma}_{K}\right| \\
h\left(\mathbf{Y}_{K-1} \mid U_{K}\right) \leq \frac{1}{2} \log (2 \pi e)^{n}\left|\mathbf{A}_{K}+\boldsymbol{\Sigma}_{K-1}\right|
\end{gathered}
$$

where (50), (51), and (52) can be obtained from (38), (34), and (44), respectively, by an appropriate change of indices. Since we have

$$
h\left(\mathbf{Y}_{K}\right) \leq \frac{1}{2} \log (2 \pi e)^{n}\left|\mathbf{S}+\boldsymbol{\Sigma}_{K}\right|
$$

from the maximum entropy theorem [11], we can get the desired bound on $R_{K}$ as follows:

$$
R_{K} \leq I\left(U_{K} ; \mathbf{Y}_{K}\right) \leq \frac{1}{2} \log \frac{\left|\mathbf{S}+\boldsymbol{\Sigma}_{K}\right|}{\left|\mathbf{A}_{K}+\boldsymbol{\Sigma}_{K}\right|} .
$$

When $K=2$ as in Section III-B, showing the existence of an $\mathbf{A}_{K}$ having the properties listed in (50)-(52) is sufficient to conclude the proof. However, when $K>2$, we need an additional tool, which is Lemma 6 , and using this tool we need to repeat this step until we are left with showing the desired bound on the first user's rate $R_{1}$. We now present the basic step that 
needs to be repeated. In particular, we now show the existence of a covariance matrix $\mathbf{A}_{K-1}$ such that

$$
\begin{aligned}
& \mathbf{J}^{-1}\left(\mathbf{X}+\mathbf{N}_{K-1} \mid U_{K-1}\right)-\boldsymbol{\Sigma}_{K-1} \preceq \mathbf{A}_{K-1} \preceq \mathbf{A}_{K} \\
& h\left(\mathbf{Y}_{K-1} \mid U_{K-1}\right)=\frac{1}{2} \log (2 \pi e)^{n}\left|\mathbf{A}_{K-1}+\boldsymbol{\Sigma}_{K-1}\right| \\
& h\left(\mathbf{Y}_{K-2} \mid U_{K-1}\right) \leq \frac{1}{2} \log (2 \pi e)^{n}\left|\mathbf{A}_{K-1}+\boldsymbol{\Sigma}_{K-2}\right| .
\end{aligned}
$$

We first note the following order

$$
\begin{aligned}
\mathbf{A}_{K} & \succeq \mathbf{J}^{-1}\left(\mathbf{X}+\mathbf{N}_{K} \mid U_{K}\right)-\boldsymbol{\Sigma}_{K} \\
& \succeq \mathbf{J}^{-1}\left(\mathbf{X}+\mathbf{N}_{K-1} \mid U_{K}\right)-\boldsymbol{\Sigma}_{K-1} \\
& \succeq \mathbf{J}^{-1}\left(\mathbf{X}+\mathbf{N}_{K-1} \mid U_{K-1}\right)-\boldsymbol{\Sigma}_{K-1}
\end{aligned}
$$

where (58) is due to (50), (59) comes from Lemma 2, and (60) follows from Lemma 6 as we can get

$$
\mathbf{J}\left(\mathbf{X}+\mathbf{N}_{K-1} \mid U_{K-1}\right) \succeq \mathbf{J}\left(\mathbf{X}+\mathbf{N}_{K-1} \mid U_{K}\right)
$$

by noting the Markov chain $U_{K} \rightarrow U_{K-1} \rightarrow \mathbf{X}+\mathbf{N}_{K-1}$.

Next, we consider the following lower bound on $h\left(\mathbf{Y}_{K-1} \mid U_{K-1}\right)$ which is due to Lemma 5

$$
\frac{1}{2} \log (2 \pi e)^{n}\left|\mathbf{J}^{-1}\left(\mathbf{X}+\mathbf{N}_{K-1} \mid U_{K-1}\right)\right| \leq h\left(\mathbf{Y}_{K-1} \mid U_{K-1}\right) .
$$

Moreover, we can get the following upper bound:

$$
\begin{aligned}
h\left(\mathbf{Y}_{K-1} \mid U_{K-1}\right) & =h\left(\mathbf{Y}_{K-1} \mid U_{K-1}, U_{K}\right) \\
& \leq h\left(\mathbf{Y}_{K-1} \mid U_{K}\right) \\
& \leq \frac{1}{2} \log (2 \pi e)^{n}\left|\mathbf{A}_{K}+\mathbf{\Sigma}_{K-1}\right|
\end{aligned}
$$

where (63) is due to the Markov chain $U_{K} \rightarrow U_{K-1} \rightarrow \mathbf{Y}_{K-1}$, (64) comes from the fact that conditioning cannot increase entropy, and (65) is due to (52).

We now define the following function:

$$
r_{K-1}(t)=\frac{1}{2} \log (2 \pi e)^{n}\left|\tilde{\mathbf{A}}_{K-1}(t)+\boldsymbol{\Sigma}_{K-1}\right|, 0 \leq t \leq 1
$$

where $\tilde{\mathbf{A}}_{K-1}(t)$ is given by

$$
\tilde{\mathbf{A}}_{K-1}(t)=(1-t)\left[\mathbf{J}^{-1}\left(\mathbf{X}+\mathbf{N}_{K-1} \mid U_{K-1}\right)-\boldsymbol{\Sigma}_{K-1}\right]+t \mathbf{A}_{K} .
$$

Using $r_{K-1}(t)$, we can recast bounds on $h\left(\mathbf{Y}_{K-1} \mid U_{K-1}\right)$ in (62) and (65) as

$$
r_{K-1}(0) \leq h\left(\mathbf{Y}_{K-1} \mid U_{K-1}\right) \leq r_{K-1}(1) .
$$

Since $r_{K-1}(t)$ is continuous in $t$ [8], due to the intermediate value theorem, there exists a $t^{*}$ such that $h\left(\mathbf{Y}_{K-1} \mid U_{K-1}\right)=$ $r_{K-1}\left(t^{*}\right)$, i.e.,

$$
h\left(\mathbf{Y}_{K-1} \mid U_{K-1}\right)=\frac{1}{2} \log (2 \pi e)^{n}\left|\mathbf{A}_{K-1}+\boldsymbol{\Sigma}_{K-1}\right|
$$

where we define $\mathbf{A}_{K-1}=\tilde{\mathbf{A}}_{K-1}\left(t^{*}\right)$. Thus, we established (56). Furthermore, it is clear that we also have (55) because of (60), (67) and $0 \leq t^{*} \leq 1$.

We now show (57). To this end, we note the following order:

$$
\begin{aligned}
\mathbf{A}_{K-1} & \succeq \mathbf{J}^{-1}\left(\mathbf{X}+\mathbf{N}_{K-1} \mid U_{K-1}\right)-\mathbf{\Sigma}_{K-1} \\
& \succeq \mathbf{J}^{-1}\left(\mathbf{X}+\mathbf{N} \mid U_{K-1}\right)-\mathbf{\Sigma}_{N}
\end{aligned}
$$

for any Gaussian $\mathbf{N}$ with covariance matrix $\boldsymbol{\Sigma}_{N} \preceq \boldsymbol{\Sigma}_{K-1}$ due to Lemma 2. The order in (71) is equivalent to

$$
\mathbf{J}\left(\mathbf{X}+\mathbf{N} \mid U_{K-1}\right) \succeq\left(\mathbf{A}_{K-1}+\boldsymbol{\Sigma}_{N}\right)^{-1}, \boldsymbol{\Sigma}_{N} \preceq \boldsymbol{\Sigma}_{K-1} .
$$

We now consider $h\left(\mathbf{Y}_{K-2} \mid U_{K-1}\right)$ as follows:

$$
\begin{aligned}
h\left(\mathbf{Y}_{K-2} \mid U_{K-1}\right) & h\left(\mathbf{Y}_{K-2} \mid U_{K-1}\right)-h\left(\mathbf{Y}_{K-1} \mid U_{K-1}\right)+h\left(\mathbf{Y}_{K-1} \mid U_{K-1}\right) \\
= & -\frac{1}{2} \int_{\boldsymbol{\Sigma}_{K-2}}^{\boldsymbol{\Sigma}_{K-1}} \mathbf{J}\left(\mathbf{X}+\mathbf{N} \mid U_{K-1}\right) d \boldsymbol{\Sigma}_{N} \\
& +\frac{1}{2} \log (2 \pi e)^{n}\left|\mathbf{A}_{K-1}+\boldsymbol{\Sigma}_{K-1}\right| \\
\leq & -\frac{1}{2} \int_{\boldsymbol{\Sigma}_{K-2}}^{\boldsymbol{\Sigma}_{K-1}}\left(\mathbf{A}_{K-1}+\boldsymbol{\Sigma}_{N}\right)^{-1} d \boldsymbol{\Sigma}_{N} \\
& +\frac{1}{2} \log (2 \pi e)^{n}\left|\mathbf{A}_{K-1}+\boldsymbol{\Sigma}_{K-1}\right| \\
= & \frac{1}{2} \log (2 \pi e)^{n}\left|\mathbf{A}_{K-1}+\boldsymbol{\Sigma}_{K-2}\right|
\end{aligned}
$$

where (74) comes from Lemma 4 and (56), and (75) is due to (72) and Lemma 3. Thus, we showed (57) as well. Also, we can establish the desired bound $R_{K-1}$ as follows

$$
\begin{aligned}
R_{K-1} & \leq I\left(U_{K-1} ; \mathbf{Y}_{K-1} \mid U_{K}\right) \\
& =h\left(\mathbf{Y}_{K-1} \mid U_{K}\right)-h\left(\mathbf{Y}_{K-1} \mid U_{K-1}\right) \\
& \leq \frac{1}{2} \log (2 \pi e)^{n}\left|\mathbf{A}_{K}+\mathbf{\Sigma}_{K-1}\right|-h\left(\mathbf{Y}_{K-1} \mid U_{K-1}\right) \\
& =\frac{1}{2} \log \frac{\left|\mathbf{A}_{K}+\mathbf{\Sigma}_{K-1}\right|}{\left|\mathbf{A}_{K-1}+\mathbf{\Sigma}_{K-1}\right|}
\end{aligned}
$$

where (78) comes from the Markov chain $U_{K} \rightarrow U_{K-1} \rightarrow Y_{K-1}$, (79) comes from (52), and (80) is due to (56).

As of now, we outlined the basic step that needs to be repeated until we are left with getting the desired bound on the first user's rate $R_{1}$. Following the analysis from (55) to (76), we can show the existence of covariance matrices $\mathbf{A}_{k}$, for $k=2, \ldots, K$, such that

$$
\begin{aligned}
\mathbf{J}^{-1}\left(\mathbf{X}+\mathbf{N}_{k} \mid U_{k}\right) & -\boldsymbol{\Sigma}_{k} \preceq \mathbf{A}_{k} \preceq \mathbf{A}_{k+1} \\
h\left(\mathbf{Y}_{k} \mid U_{k}\right) & =\frac{1}{2} \log (2 \pi e)^{n}\left|\mathbf{A}_{k}+\boldsymbol{\Sigma}_{k}\right| \\
h\left(\mathbf{Y}_{k-1} \mid U_{k}\right) & \leq \frac{1}{2} \log (2 \pi e)^{n}\left|\mathbf{A}_{k}+\boldsymbol{\Sigma}_{k-1}\right|
\end{aligned}
$$

where we set $\mathbf{A}_{K+1}=\mathbf{S}$. Using these relations, we can get the bound on $R_{k}$ for any $k=2, \ldots, K$, as

$$
\begin{aligned}
R_{k} & \leq I\left(U_{k} ; \mathbf{Y}_{k} \mid U_{k+1}\right) \\
& =h\left(\mathbf{Y}_{k} \mid U_{k+1}\right)-h\left(\mathbf{Y}_{k} \mid U_{k}\right) \\
& \leq \frac{1}{2} \log (2 \pi e)^{n}\left|\mathbf{A}_{k+1}+\boldsymbol{\Sigma}_{k}\right|-h\left(\mathbf{Y}_{k} \mid U_{k}\right) \\
& =\frac{1}{2} \log \frac{\left|\mathbf{A}_{k+1}+\boldsymbol{\Sigma}_{k}\right|}{\left|\mathbf{A}_{k}+\boldsymbol{\Sigma}_{k}\right|}
\end{aligned}
$$

where we set $U_{K+1}=\phi$. The equality in (85) comes from the Markov chain $U_{k+1} \rightarrow U_{k} \rightarrow \mathbf{Y}_{k}$, (86) is obtained by using 
(83), and (87) is due to (82). For $k=1$, we can get the bound on $R_{1}$ as

$$
\begin{aligned}
R_{1} & \leq I\left(\mathbf{X} ; \mathbf{Y}_{1} \mid U_{2}\right) \\
& =h\left(\mathbf{Y}_{1} \mid U_{2}\right)-\frac{1}{2} \log (2 \pi e)^{n}\left|\mathbf{\Sigma}_{1}\right| \\
& \leq \frac{1}{2} \log \frac{\left|\mathbf{A}_{2}+\mathbf{\Sigma}_{1}\right|}{\left|\boldsymbol{\Sigma}_{1}\right|}
\end{aligned}
$$

where (90) comes from (83). Finally, we define $\mathbf{K}_{k}=\mathbf{A}_{k+1}$ $\mathbf{A}_{k}$ for $k=1, \ldots, K$ where $\mathbf{A}_{1}=\phi, \mathbf{A}_{K+1}=\mathbf{S}$, and plug these into (87)-(90) which yields the expressions in Theorem 1.

\section{CONCLUSIONS}

We provide an alternative proof for the capacity region of the degraded Gaussian MIMO broadcast channel. As opposed to the existing proofs in [1] and [2], our proof does not use the channel enhancement technique [1]. Our proof starts with the single-letter description of the capacity region of the degraded broadcast channel, and by using it, obtains a tight (i.e., achievable) outer bound for the capacity region of the degraded Gaussian MIMO broadcast channel, by using two main technical tools. The first one is the generalized de Bruijn identity that gives a connection between the differential entropy and the Fisher information [5]. The second one is an inequality due to [6] and [7] that lower bounds the differential entropy in terms of the Fisher information matrix.

\section{APPENDIX}

\section{PROOF OF LEMMA 5}

We define the function $f(\epsilon)$ as follows:

$$
f(\epsilon)=h(\mathbf{X}+\sqrt{\epsilon} \mathbf{N} \mid U)-\frac{1}{2} \log \left|(2 \pi e)\left(\mathbf{J}^{-1}(\mathbf{X} \mid U)+\epsilon \mathbf{\Sigma}\right)\right|
$$

where $\epsilon \geq 0$.

We need to prove that $f(0) \geq 0$. We will show that $f(\epsilon)$ is monotonically decreasing in $\epsilon$, and that $\lim _{\epsilon \rightarrow \infty} f(\epsilon)=0$. This will prove $f(0) \geq 0$. To this end, we introduce the following lemma, which will be used subsequently.

Lemma 7 ([8, Corollary 4]): Let X, Y, U be length- $n$ random vectors and let the density for any combination of these random vectors exist. Moreover, let $\mathbf{X}$ and $\mathbf{Y}$ be conditionally independent given $\mathbf{U}$. Then, we have

$$
\mathbf{J}(\mathbf{X}+\mathbf{Y} \mid \mathbf{U}) \preceq\left[\mathbf{J}(\mathbf{X} \mid \mathbf{U})^{-1}+\mathbf{J}(\mathbf{Y} \mid \mathbf{U})^{-1}\right]^{-1} .
$$

Fix $\epsilon_{1}, \epsilon_{2}$ such that $0<\epsilon_{1} \leq \epsilon_{2}$. Using Lemma 4, we have

$$
\begin{aligned}
h\left(\mathbf{X}+\sqrt{\epsilon_{2}} \mathbf{N} \mid U\right)-h( & \left.\mathbf{X}+\sqrt{\epsilon_{1}} \mathbf{N} \mid U\right) \\
& =\frac{1}{2} \int_{\epsilon_{1} \boldsymbol{\Sigma}}^{\epsilon_{2} \boldsymbol{\Sigma}} \mathbf{J}(\mathbf{X}+\mathbf{T} \mid U) d \boldsymbol{\Sigma}_{T}
\end{aligned}
$$

where $\mathbf{T}$ is a Gaussian random vector with covariance matrix $\boldsymbol{\Sigma}_{T}$ such that $\epsilon_{1} \boldsymbol{\Sigma} \preceq \boldsymbol{\Sigma}_{T} \preceq \epsilon_{2} \boldsymbol{\Sigma}$, and independent of $(U, \mathbf{X})$. Using Lemma 7 in conjunction with Lemma 1, we get

$$
\mathbf{J}(\mathbf{X}+\mathbf{T} \mid U) \preceq\left[\mathbf{J}^{-1}(\mathbf{X} \mid U)+\mathbf{\Sigma}_{T}\right]^{-1} .
$$

Plugging (94) into (93) and invoking Lemma 3, we get

$$
\begin{aligned}
& h\left(\mathbf{X}+\sqrt{\epsilon_{2}} \mathbf{N} \mid U\right)-h\left(\mathbf{X}+\sqrt{\epsilon_{1}} \mathbf{N} \mid U\right) \\
& \leq \frac{1}{2} \log \frac{\left|(2 \pi e)\left(\mathbf{J}^{-1}(\mathbf{X} \mid U)+\epsilon_{2} \boldsymbol{\Sigma}\right)\right|}{\left|(2 \pi e)\left(\mathbf{J}^{-1}(\mathbf{X} \mid U)+\epsilon_{1} \boldsymbol{\Sigma}\right)\right|} .
\end{aligned}
$$

Rearranging (95) yields

$$
f\left(\epsilon_{2}\right) \leq f\left(\epsilon_{1}\right), \quad \epsilon_{1} \leq \epsilon_{2}
$$

which proves that $f(\epsilon)$ is monotonically decreasing in $\epsilon$.

We now consider upper and lower bounds on $f(\epsilon)$. We have the following upper bound on $f(\epsilon)$ :

$$
\begin{aligned}
f(\epsilon) & =h(\mathbf{X}+\sqrt{\epsilon} \mathbf{N} \mid U)-\frac{1}{2} \log \left|(2 \pi e)\left(\mathbf{J}^{-1}(\mathbf{X} \mid U)+\epsilon \boldsymbol{\Sigma}\right)\right| \\
& \leq \frac{1}{2} \log \frac{|\mathbf{K}+\epsilon \boldsymbol{\Sigma}|}{\left|\mathbf{J}^{-1}(\mathbf{X} \mid U)+\epsilon \boldsymbol{\Sigma}\right|} \\
& =\frac{1}{2} \log \frac{\left|\boldsymbol{\Sigma}^{-1 / 2} \mathbf{K} \boldsymbol{\Sigma}^{-1 / 2}+\epsilon \mathbf{I}\right|}{\left|\boldsymbol{\Sigma}^{-1 / 2} \mathbf{J}^{-1}(\mathbf{X} \mid U) \mathbf{\Sigma}^{-1 / 2}+\epsilon \mathbf{I}\right|} \\
& =\frac{1}{2} \log \prod_{i=1}^{n} \frac{\tilde{\lambda}_{i}+\epsilon}{\lambda_{i}+\epsilon}
\end{aligned}
$$

where (98) comes from the maximum entropy theorem [11] and $\mathbf{K}$ denotes the covariance matrix of $\mathbf{X}$. In (100), we denote the eigenvalues of $\boldsymbol{\Sigma}^{-1 / 2} \mathbf{K} \boldsymbol{\Sigma}^{-1 / 2}$ with $\left\{\tilde{\lambda}_{i}\right\}_{i=1}^{n}$, and of $\boldsymbol{\Sigma}^{-1 / 2} \mathbf{J}^{-1}(\mathbf{X} \mid U) \boldsymbol{\Sigma}^{-1 / 2}$ with $\left\{\lambda_{i}\right\}_{i=1}^{n}$. Furthermore, we have the following lower bound on $f(\epsilon)$ :

$$
f(\epsilon)=h(\mathbf{X}+\sqrt{\epsilon} \mathbf{N} \mid U)-\frac{1}{2} \log \left|(2 \pi e)\left(\mathbf{J}^{-1}(\mathbf{X} \mid U)+\epsilon \boldsymbol{\Sigma}\right)\right|
$$

$$
\begin{aligned}
& \geq \frac{1}{2} \log \frac{|\epsilon \boldsymbol{\Sigma}|}{\left|\mathbf{J}^{-1}(\mathbf{X} \mid U)+\epsilon \boldsymbol{\Sigma}\right|} \\
& =\frac{1}{2} \log \frac{\epsilon^{n}}{\left|\boldsymbol{\Sigma}^{-1 / 2} \mathbf{J}^{-1}(\mathbf{X} \mid U) \boldsymbol{\Sigma}^{-1 / 2}+\epsilon \mathbf{I}\right|} \\
& =\frac{1}{2} \log \prod_{i=1}^{n} \frac{\epsilon}{\lambda_{i}+\epsilon}
\end{aligned}
$$

where (102) comes from the fact that conditioning cannot increase entropy, and in (104), we denote the eigenvalues of $\boldsymbol{\Sigma}^{-1 / 2} \mathbf{J}^{-1}(\mathbf{X} \mid U) \boldsymbol{\Sigma}^{-1 / 2}$ with $\left\{\lambda_{i}\right\}_{i=1}^{n}$. Comparison of (100) and (104) yields

$$
\frac{1}{2} \log \prod_{i=1}^{n} \frac{\epsilon}{\lambda_{i}+\epsilon} \leq f(\epsilon) \leq \frac{1}{2} \log \prod_{i=1}^{n} \frac{\tilde{\lambda}_{i}+\epsilon}{\lambda_{i}+\epsilon} .
$$

Taking the limit as $\epsilon \rightarrow \infty$ yields $\lim _{\epsilon} \rightarrow \infty f(\epsilon)=0$. Combining this with the fact that $f(\epsilon)$ decreases monotonically in $\epsilon$ yields $f(0) \geq 0$, and consequently,

$$
h(\mathbf{X} \mid U) \geq \frac{1}{2} \log (2 \pi e)^{n}\left|\mathbf{J}^{-1}(\mathbf{X} \mid U)\right|,
$$

completing the proof.

\section{REFERENCES}

[1] H. Weingarten, Y. Steinberg, and S. Shamai (Shitz), "The capacity region of the Gaussian multiple-input multiple-output broadcast channel," IEEE Trans. Inf. Theory, vol. 52, no. 9, pp. 3936-3964, Sep. 2006.

[2] T. Liu and P. Viswanath, "An extremal inequality motivated by multiterminal information theoretic problems," IEEE Trans. Inf. Theory, vol. 53, no. 5, pp. 1839-1851, May 2007. 
[3] P. Bergmans, "A simple converse for broadcast channels with additive white Gaussian noise," IEEE Trans. Inf. Theory, vol. 20, no. 3, pp. 279-280, Mar. 1974.

[4] K. Marton, "A coding theorem for the discrete memoryless channels," IEEE Trans. Inf. Theory, vol. 25, no. 1, pp. 306-311, May 1979.

[5] D. P. Palomar and S. Verdu, "Gradient of mutual information in linear vector Gaussian channels," IEEE Trans. Inf. Theory, vol. 52, no. 1, pp. 141-154, Jan. 2006.

[6] A. Dembo, "Information inequalities and uncertainty principles," Dept. Stat., Stanford Univ., Stanford, CA, Tech. Rep. 75, 1990.

[7] A. Dembo, T. M. Cover, and J. A. Thomas, "Information theoretic inequalities," IEEE Trans. Inf. Theory, vol. 37, no. 6, pp. 1501-1518, Nov. 1991.

[8] E. Ekrem and S. Ulukus, "The secrecy capacity region of the Gaussian MIMO multi-receiver wiretap channel," IEEE Trans. Inf. Theory, vol. 57, no. 4, pp. 2083-2114, Apr. 2011.

[9] E. Ekrem and S. Ulukus, "Degraded compound multi-receiver wiretap channels," IEEE Trans. Inf. Theory, Oct. 2009, submitted for publication.

[10] H. Weingarten, T. Liu, S. Shamai, Y. Steinberg, and P. Viswanath, “The capacity region of the degraded multiple-input multiple-output compound broadcast channel," IEEE Trans. Inf. Theory, vol. 55, no. 11, pp. 5011-5023, Nov. 2009.

[11] T. Cover and J. Thomas, Elements of Information Theory, 2nd ed. Hoboken, NJ: Wiley, 2006.

[12] A. J. Stam, "Some inequalities satisfied by the quantities of information of Fisher and Shannon," Inf. Control, vol. 2, pp. 101-112, Jun. 1959

[13] N. M. Blachman, "The convolution inequality for entropy powers," IEEE Trans. Inf. Theory, vol. IT-11, no. 2, pp. 267-271, Apr. 1965.

Ersen Ekrem (S'08) received the B.S. and M.S. degrees in electrical and electronics engineering from Bogaziçi University, Istanbul, Turkey, in 2006 and 2007 , respectively. Currently, he is working toward the Ph.D. degree in the department of electrical and computer engineering at the University of Maryland, College Park.

His research interests include information theory and wireless communications.
Sennur Ulukus (S'90-M'98) is a Professor of Electrical and Computer Engineering at the University of Maryland at College Park, where she also holds a joint appointment with the Institute for Systems Research (ISR). Prior to joining UMD, she was a Senior Technical Staff Member at AT\&T Labs-Research. She received her Ph.D. degree in Electrical and Computer Engineering from Wireless Information Network Laboratory (WINLAB), Rutgers University, and B.S. and M.S. degrees in Electrical and Electronics Engineering from Bilkent University. Her research interests are in wireless communication theory and networking, network information theory for wireless communications, signal processing for wireless communications, information-theoretic physical-layer security, and energy-harvesting communications.

Dr. Ulukus received the 2003 IEEE Marconi Prize Paper Award in Wireless Communications, the 2005 NSF CAREER Award, and the 2010-2011 ISR Outstanding Systems Engineering Faculty Award. She served as an Associate Editor for the IEEE TRANSACTIONS ON INFORMATION THEORY between 2007-2010, as an Associate Editor for the IEEE TRANSACTIONS ON COMMUNICATIONS between 2003-2007, as a Guest Editor for the Journal of Communications and Networks for the special issue on energy harvesting in wireless networks, as a Guest Editor for the IEEE TRANSACTIONS ON INFORMATION THEORY for the special issue on interference networks, as a Guest Editor for the IEEE JOURNAL on SELECTEd AREas IN COMMUNICATIONS for the special issue on multiuser detection for advanced communication systems and networks. She served as the TPC co-chair of the Communication Theory Symposium at the 2007 IEEE Global Telecommunications Conference, the Medium Access Control (MAC) Track at the 2008 IEEE Wireless Communications and Networking Conference, the Wireless Communications Symposium at the 2010 IEEE International Conference on Communications, the 2011 Communication Theory Workshop, the Physical-Layer Security Workshop at the 2011 IEEE International Conference on Communications, the Physical-Layer Security Workshop at the 2011 IEEE Global Telecommunications Conference. She was the Secretary of the IEEE Communication Theory Technical Committee (CTTC) in 2007-2009. 